\title{
Zum Inhalt von Band VI
}

Der vorliegende sechste und letzte Band „Statistik, Festkörper, Materialien“ ist den großen, makroskopischen Vielteilchensystemen gewidmet. Die thermodynamischen Parameter dieser Systeme werden als statistische Größen des Verhaltens der einzelnen Teilchen des Systems berechenbar. Hier wird die klassische Maxwell-Boltzmann Statistik mit den Quantenstatistiken nach Fermi-Dirac und Bose-Einstein verglichen. Die Festkörperphysik beschäftigt sich mit großen Systemen, sie ist eine Physik der Kristalle. Den Einstieg dafür bilden daher die Kristallbindungen, die Kristallstruktur und der reziproke Raum. Bei der Erklärung der spezifischen Wärme muss wieder die klassische Physik verlassen werden: Die Eigenschwingungen des Kristallgitters werden durch Quasiteilchen, die Phononen, beschrieben. Das Verhalten der Elektronen im Festkörper nimmt einen wesentlichen Platz ein. Viele Bereiche der aktuellen physikalischen Forschung mit großer Bedeutung für die technische Anwendung haben hier ihren Ausgangspunkt. Die Physik nichtkristalliner Materialien bildet den Abschluss dieses Bandes. Hier geht es um amorphe Festkörper, Flüssigkristalle, Quasikristalle, Formgedächtnis-Legierungen und Nanomaterialien. 
